Research Article

\title{
Multiple Lump Solutions of the $(4+1)$-Dimensional Fokas Equation
}

\author{
Hongcai Ma $\mathbb{D}^{1,2}$ Yunxiang Bai $\mathbb{D},{ }^{1,2}$ and Aiping Deng ${ }^{1,2}$ \\ ${ }^{1}$ Department of Applied Mathematics, Donghua University, Shanghai 201620, China \\ ${ }^{2}$ Institute for Nonlinear Sciences, Donghua University, Shanghai 201620, China \\ Correspondence should be addressed to Hongcai Ma; hongcaima@hotmail.com and Yunxiang Bai; baiyunxiang429@163.com
}

Received 13 March 2020; Revised 6 May 2020; Accepted 11 May 2020; Published 10 June 2020

Academic Editor: Ming Mei

Copyright (C) 2020 Hongcai Ma et al. This is an open access article distributed under the Creative Commons Attribution License, which permits unrestricted use, distribution, and reproduction in any medium, provided the original work is properly cited.

In this paper, we investigate multiple lump wave solutions of the new $(4+1)$-dimensional Fokas equation by adopting a symbolic computation method. We get its 1-lump solutions, 3-lump solutions, and 6-lump solutions by using its bilinear form. Moreover, some basic characters and structural features of multiple lump waves are explained by depicting the three-dimensional plots.

\section{Introduction}

Nonlinear evolution equations can be used to simulate many nonlinear phenomena in the real world, which appear in many areas, especially in physical [1], engineering sciences [2], applied mathematics [3], chemistry, and biology [4]. Recently, it is well known that rogue waves play an essential role in helping us apprehending the qualitative properties of many phenomena; it is interesting that lump functions can provide approximate fitting prototypes to model rogue waves. In addition to appearing in the ocean [5], lump waves also actually appear in many other fields, such as atmosphere [6], superfluids [7], and capillary waves [8]. Further study of lump waves will help us interpret some unknown fields more deeply. Certain ways have been arranged to solve the lump wave solutions of some equations; they are inclusive of the Hirota bilinear method $[9,10]$, the inverse scattering transformation [11], the Darboux transformation [12], the Bäcklund transformation [13], the functional variable method [14], the reduced differential transform method [15], and so on. Many integrable equations which have lump wave solutions are enumerated here, for example, the $(3+1)$-dimensional KPI equation [16], the Davey-Stewartson I equation [17], the $(3+1)$-dimensional nonlinear evolution equation
$[18,19]$, and the nonlinear Schrödinger equation [20]. Generally speaking, it is easier to solve the lower order rational solutions than to solve the multiple lump waves of the nonlinear evolution equation. In this paper, we mainly work on a $(4+1)$-dimensional Fokas equation.

$$
4 u_{t x}-u_{x x x y}+u_{x y y y}+12 u_{x} u_{y}+12 u u_{x y}-6 u_{z w}=0 \text {, }
$$

which was first derived by Fokas by the generalization of two critical nonlinear evolution equations, which are the integrable KP equation and DS equation [21]. The $(4+1)$-dimensional Fokas equation could be applied to portray nonelastic and elastic interactions [21, 22]. In nonlinear wave theory, KP and DS equations can be used to characterize the surface waves and internal waves in straits or channels of varying depth and width, respectively [23-26]. The significance of the $(4+1)$-dimensional Fokas equation follows naturally from the physical applications of the KP and DS equations. Therefore, the $(4+1)$-dimensional Fokas equation could be adopted to represent a number of phenomena in fluid mechanics, optical fiber communications, ocean engineering, and many others. More recently, $(4+1)$-dimensional Fokas equation has been discussed by some scholars. Demiray et al. obtained the exact solutions of Equation (1) by applying 
the generalized Kudryashov method (GKM) [27]. Based on the Hirota bilinear form, two classes of lump-type solutions of Equation (1) are studied by Cheng and Zhang [28]. In 2017, two distinct methods, namely, the modified simple equation method (MSEM) and the extended simplest equation method (ESEM), are employed to look for exact traveling wave solutions of Equation (1) [29]. Zhang et al. used the fractional subequation method to obtain the exact analytical solutions of Equation (1) [30]. The lump-bell solutions of Equation (1) are obtained based on the bilinear equation and different test functions in [31]. Particularly, El-Ganaini and $\mathrm{Al}$-Amr discussed the space-time fractional $(4+1)$-dimensional Fokas equation via the functional variable, the generalized Kudryashov, the Jacobi elliptic function expansion, and the generalized Riccati equation mapping methods and got abundant distinct types of new exact solutions [32]. Zhang and Xia obtained soliton solutions, fissionable wave solutions, M-lump solutions, and interaction solutions of the $(4+1)$-dimensional Fokas equation based on the Hirota bilinear method [33]. However, Zhaqilao proposed a novel method to construct the multiple rogue wave solutions of nonlinear partial differential equation; the multiple rogue wave solutions of Equation (1) have not been extracted by this new method. This paper is constructed as follows. In Section 2, the bilinear equation of Equation (1) is acquired. The 1-lump waves are also gained by employing a new ansatz. In Section 3, 3-lump waves of Equation ((1)) are researched when the subscript of $f_{n}$ is equal to 1 in Equation (10). In Section 4, the 6lump waves of Equation (1) are studied when the subscript of $f_{n}$ is equal to 2 in Equation (10). Section 5 is devoted to a short conclusion and discussion.

\section{1-Lump Solutions}

The fundamental desire of this section is to investigate the 1lump solutions of the new $(4+1)$-dimensional Fokas equation. Firstly, setting $X=x+m y+n t$ and $Z=z+c w$ in Equation (1) yields

$$
4 n u_{X X}+\left(m^{3}-m\right) u_{X X X X}+12 m u_{X}^{2}+12 m u u_{X X}-6 c u_{Z Z}=0,
$$

where $m, n, c$ are all real parameters. With the help of variable transformation

$$
u=u_{0}+\left(m^{2}-1\right)(\ln f)_{X X},
$$

we can convert the Equation (2) into a bilinear form that reads

$$
\left(4 n D_{X}^{2}+\left(m^{3}-m\right) D_{X}^{4}-6 c D_{Z}^{2}\right) f \cdot f=0,
$$

where $\quad D_{X}^{2} f \cdot f=f f_{X X}-f_{X}^{2}, \quad D_{X}^{4} f \cdot f=3 f_{X X}^{2}-4 f_{X} f_{X X X}+f$ $f_{X X X X}$, and $D_{Z}^{2} f \cdot f=f f_{Z Z}-f_{Z}^{2}$, where $f$ is a real function with regard to variable $X, Z . D_{X}^{2}, D_{X}^{4}$, and $D_{Z}^{2}$ are called Hirota bilinear $\mathrm{D}$ operators. By applying the symbolic computation approach, assuming

$$
f=(X-\beta)^{2}+a_{1}(Z-\alpha)^{2}+a_{0}
$$

where $\alpha, \beta, a_{1}$, and $a_{0}$ are constants to be determined.

Substituting (5) into (4) and equating the coefficients of all powers of $X^{i_{1}} Z^{i_{2}}$ to 0 , one has

$$
\begin{aligned}
& 12 c a_{1}^{2}+8 m a_{1}=0, \\
&-24 c \alpha a_{1}^{2}-16 m \alpha a_{1}=0, \\
&-12 c a_{1}-8 m=0, 24 \beta c a_{1}+16 \beta m=0, \\
& 12 a_{1}^{2} \alpha^{2} c+\left(\left(-12 \beta^{2}-12 a_{0}\right) c+8 m \alpha^{2}\right) a_{1} \\
&+\left(-8 \beta^{2}+8 a_{0}\right) m+12 n^{3}-12 n=0 .
\end{aligned}
$$

Solving these equations, one has

$$
\begin{aligned}
& a_{0}=-\frac{3 n\left(n^{2}-1\right)}{4 m}, \\
& a_{1}=-\frac{2 m}{3 c} .
\end{aligned}
$$

Therefore, we can get a solution of Equation (4) as

$$
f=(X-\beta)^{2}-\frac{2 m(Z-\alpha)^{2}}{3 c}-\frac{3 n\left(n^{2}-1\right)}{4 m} .
$$

By using variable transformation (3), the 1-lump wave solutions of Equation (1) read

$$
u(x, y, z, t, w)=\left(m^{2}-1\right)\left(\frac{2}{(X-\beta)^{2}-\left(\left(2 m(Z-\alpha)^{2}\right) / 3 c\right)-\left(\left(3 n\left(n^{2}-1\right)\right) / 4 m\right)}-\frac{(2 X-2 \beta)^{2}}{\left((X-\beta)^{2}-\left(\left(2 m(Z-\alpha)^{2}\right) / 3 c\right)-\left(\left(3 n\left(n^{2}-1\right)\right) / 4 m\right)\right)^{2}}\right)
$$

where $X=x+m t, Z=z+c w, m, n, c, \alpha, \beta$ are arbitrary real constants. The 1-lump wave (9) has the structure for three wave peaks. One peak is higher than the water level, and the other two are opposite. Figure 1 presents the three dimensional plot, the density plot, and the corresponding contour plot of the 1-lump wave solution of Equation (1). From 


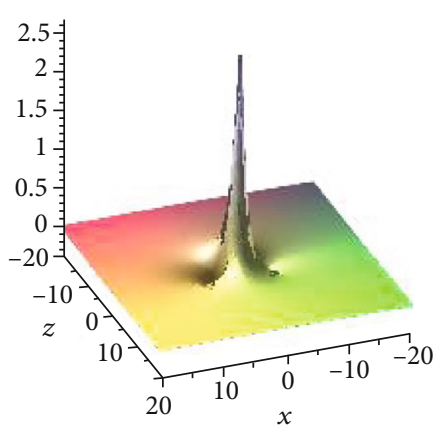

(a)

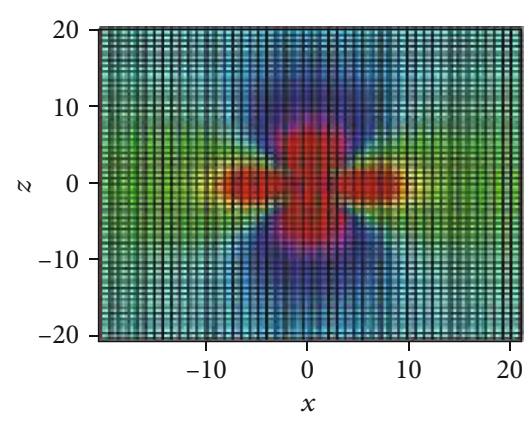

(b)

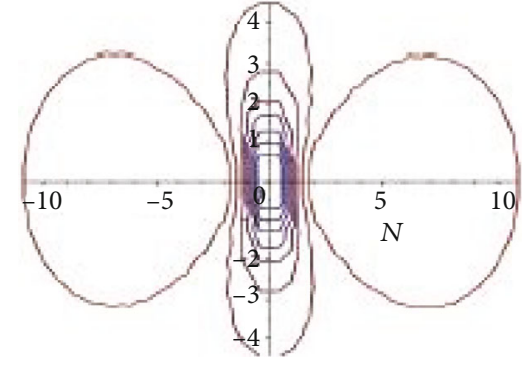

(c)

FIgUre 1: The 1-lump solution $u$ of Equation (1) with the parameter selections $m=-2, n=2, c=1, u_{0}=0, \alpha=0, \beta=0$. (a) Perspective view of the wave $u(X, Z)$, (b) overhead view of the wave $u(X, Z)$, and (c) the corresponding contour plot $u(X, Z)$.

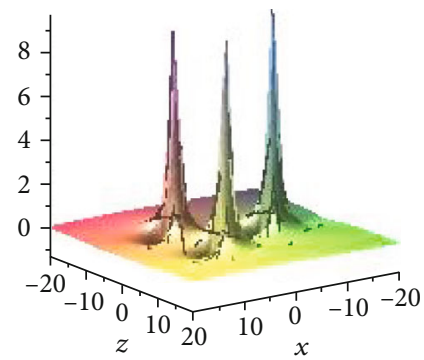

(a)

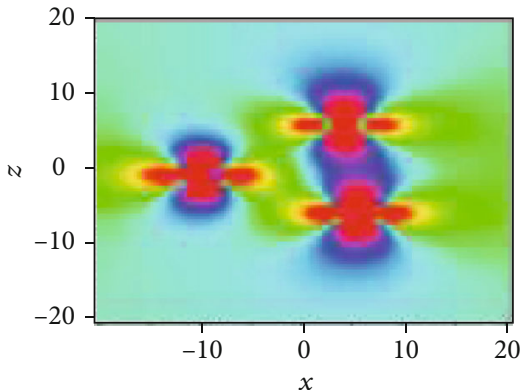

(b)

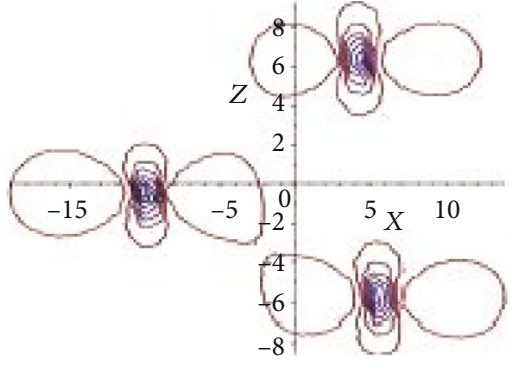

(c)

Figure 2: The 3-lump solution $u$ of Equation (1) with the parameter selections $m=-3, n=2, c=1, u_{0}=0, \alpha=1000, \beta=1000, c_{2,0}=1$, $b_{0,2}=1$. (a) Perspective view of the wave $u(X, Z)$, (b) overhead view of the wave $u(X, Z)$, and (c) the corresponding contour plot $u(X, Z)$.

Figure 1, we can see that the 1-lump wave has one center $(\beta, \alpha)$. Furthermore, at the point $\left(\left(\left(\sqrt{-3 m n\left(n^{2}-1\right)}+2 m \beta\right) / 2 m\right)\right.$, $\alpha)$ in the plane $(X, Z)$, the maximum amplitude of the 1lump wave is $\left(u_{0}-\left(\left(2 \sqrt{-3 m n\left(n^{2}-1\right)}\right) /\left(3 n\left(n^{2}-1\right)\right)\right)\right)$.

\section{3-Lump Solutions}

In the section, in order to construct the multiple lump solutions, we propose the notation just like this:

$$
\begin{aligned}
F_{n}(X, Z)= & \sum_{k=0}^{n(n+1) / 2} \sum_{i=0}^{k} a_{n(n+1)-2 k, 2 i} Z^{2 i} X^{n(n+1)-2 k}, \\
P_{n}(X, Z)= & \sum_{k=0}^{n(n+1) / 2} \sum_{i=0}^{k} b_{n(n+1)-2 k, 2 i} X^{2 i} Z^{n(n+1)-2 k}, \\
Q_{n}(X, Z)= & \sum_{k=0}^{n(n+1) / 2} \sum_{i=0}^{k} c_{n(n+1)-2 k, 2 i} Z^{2 i} X^{n(n+1)-2 k}, \\
f(X, Z)= & f_{n+1}(X, Z)=F_{n+1}(X, Z)+2 \alpha Z P_{n}(X, Z) \\
& +2 \beta X Q_{n}(X, Z)+\left(\alpha^{2}+\beta^{2}\right),
\end{aligned}
$$

where $a_{i, j}, b_{i, j}$, and $c_{i, j}$ are arbitrary constants. Then, we take $n=1$,

$$
\begin{aligned}
f(X, Z)= & F_{2}(X, Z)+2 \alpha Z P_{1}(X, Z) \\
& +2 \beta X Q_{1}(X, Z)+\left(\alpha^{2}+\beta^{2}\right),
\end{aligned}
$$

where

$$
\begin{aligned}
F_{2}(X, Z)= & X^{6}+a_{4,0} X^{4}+a_{4,2} Z^{2} X^{4} \\
& +\left(a_{2,0}+a_{2,2} Z^{2}+a_{2,4} Z^{4}\right) X^{2} \\
& +a_{0,0}+a_{0,2} Z^{2}+a_{0,4} Z^{4}+a_{0,6} Z^{6}, \\
P_{1}(X, Z)= & b_{0,0}+b_{0,2} X^{2}+b_{2,0} Z^{2}, \\
Q_{1}(X, Z)= & c_{0,0}+c_{0,2} Z^{2}+c_{2,0} X^{2} .
\end{aligned}
$$

Substituting Equation (11) into Equation (4) and collecting all the coefficients of $X^{i_{1}} Z^{i_{2}}$, we can get a group of constraining relationships for the parameters. Dealing with these equations, one gets 


$$
\begin{aligned}
& a_{0,0}=-\frac{5625 n^{9}-192 \beta^{2} c_{2,0}^{2} m^{3}-16875 n^{7}+32 \alpha^{2} c m^{2} b_{0,2}^{2}+192 \alpha^{2} m^{3}+192 \beta^{2} m^{3}+16875 n^{5}-5625 n^{3}}{192 m^{3}}, \\
& a_{0,2}=-\frac{475 n^{2}\left(n^{4}-2 n^{2}+1\right)}{24 c m}, \\
& a_{0,4}=-\frac{17 n\left(n^{2}-1\right) m}{9 c^{2}} \\
& a_{0,6}=-\frac{8 m^{3}}{27 c^{3}} \\
& a_{2,0}=-\frac{125 n^{2}\left(n^{4}-2 n^{2}+1\right)}{16 m^{2}}, \\
& a_{2,2}=\frac{15 n\left(n^{2}-1\right)}{c} \text {, } \\
& a_{2,4}=\frac{4 m^{2}}{3 c^{2}}, \\
& a_{4,0}=-\frac{-25 n\left(n^{2}-1\right)}{4 m}, \\
& a_{4,2}=-\frac{2 m}{c}, \\
& b_{0,0}=-\frac{5 b_{0,2} n\left(n^{2}-1\right)}{12 m}, \\
& b_{2,0}=\frac{2 m b_{0,2}}{9 c}, \\
& c_{0,0}=\frac{c_{2,0} n\left(n^{2}-1\right)}{4 m}, \\
& c_{0,2}=\frac{2 m c_{2,0}}{c}, \\
& c_{2,0}=c_{2,0} \text {, } \\
& b_{0,2}=b_{0,2} \text {, }
\end{aligned}
$$

where $b_{0,2}$ and $c_{2,0}$ are arbitrary constants. Thus, the 3-lump solution of Equation (1) is shown by

$$
u=u_{0}+\left(m^{2}-1\right)(\ln f)_{X X}
$$

where $f$ is given in Equation (11), in which $X=x+m y+n t$, $Z=z+c w$. By increasing the value of $\alpha$ and $\beta$, 3-lump wave merge and their centers form a triangle (see Figure 2 ). The 3-lump wave is the arrangement of three 1-lump waves in the plane $(X, Z)$.

\section{6-Lump Solutions}

To get the 6-lump solution of Equation (1), the 6-lump solution waves of a $(4+1)$-dimensional Fokas equation can be presented if we choose $n=2$,

$$
\begin{aligned}
f(X, Z)= & F_{3}(X, Z)+2 \alpha Z P_{2}(X, Z)+2 \beta X Q_{2}(X, Z) \\
& +\left(\alpha^{2}+\beta^{2}\right) F_{1}(X, Z),
\end{aligned}
$$

where

$$
\begin{aligned}
F_{3}(X, Z)= & X^{12}+\left(a_{10,0}+a_{10,2} Z^{2}\right) X^{10} \\
& +\left(a_{8,0}+a_{8,2} Z^{2}+a_{8,4} Z^{4}\right) X^{8} \\
& +\left(a_{6,0}+a_{6,2} Z^{2}+a_{6,4} Z^{4}+a_{6,6} Z^{6}\right) X^{6} \\
& +\left(a_{4,0}+a_{4,2} Z^{2}+a_{4,4} Z^{4}+a_{4,6} Z^{6}+a_{4,8} Z^{8}\right) X^{4} \\
& +\left(a_{2,0}+a_{2,2} Z^{2}+a_{2,4} Z^{4}+a_{2,6} Z^{6}+a_{2,8} Z^{8}+a_{2,10} Z^{10}\right) X^{2} \\
& +a_{0,0}+a_{0,2} Z^{2}+a_{0,4} Z^{4}+a_{0,6} Z^{6} \\
& +a_{0,8} Z^{8}+a_{0,10} Z^{10}+a_{0,12} Z^{12},
\end{aligned}
$$




$$
\begin{aligned}
P_{2}(X, Z)= & b_{0,0}+\left(b_{2,0}+b_{2,2} X^{2}+b_{2,4} X^{4}\right) Z^{2} \\
& +\left(b_{4,0}+b_{4,2} X^{2}\right) Z^{4}+Z^{6}+b_{0,2} X^{2} \\
& +b_{0,4} X^{4}+b_{0,6} X^{6} \\
Q_{2}(X, Z)= & c_{0,0}+c_{0,2} Z^{2}+c_{0,4} Z^{4}+c_{0,6} Z^{6} \\
& +\left(c_{2,0}+c_{2,2} Z^{2}+c_{2,4} Z^{4}\right) X^{2} \\
& +\left(c_{4,0}+c_{4,2} Z^{2}\right) X^{4}+X^{6} \\
F_{1}(X, Z)= & X^{2}+a_{0,2} Z^{2}+a_{0,0} .
\end{aligned}
$$

Substitute (15) into (4) and let all the coefficients of the different powers of $X^{i_{1}} Z^{i_{2}}$ equal to zero, we can get a set of constraining relations for the parameters. Figuring out these equations, one has

$$
\begin{aligned}
& a_{0,0}=\frac{1}{36864 m^{8}\left(\alpha^{2}+\beta^{2}+1\right)}\left(\left(878826025 m^{2} n^{17}\right.\right. \\
& -5272956150 m^{2} n^{15}+13182390375 m^{2} n^{13} \\
& -17576520500 m^{2} n^{11}+472392 \alpha^{2} c^{7} n^{2} \\
& -27648 \beta^{2} m^{7} n^{2}+13182390375 m^{2} n^{9}-472392 \alpha^{2} c^{7} \\
& \left.\left.+27648 m^{7} \beta^{2}-5272956150 m^{2} n^{7}+878826025 m^{2} n^{5}\right) n\right) \text {, } \\
& a_{0,10}=\frac{464 m^{4} n\left(n^{2}-1\right)}{243 c^{5}}, \\
& a_{0,12}=\frac{64 m^{6}}{729 c^{6}} \\
& a_{0,2}=\frac{1}{2304 m^{6} c\left(\alpha^{2}+\beta^{2}+1\right)} \\
& \cdot\left(150448375 m^{2} n^{15}-752241875 m^{2} n^{13}\right. \\
& +1504483750 m^{2} n^{11}-1504483750 n^{9} m^{2} \\
& +26244 \alpha^{2} c^{7}-1536 m^{7} \beta^{2}+752241875 m^{2} n^{7} \\
& \left.-150448375 m^{2} n^{5}\right) \text {, } \\
& a_{0,4}=\frac{16391725 n^{4}\left(n^{8}-4 n^{6}+6 n^{4}-4 n^{2}+1\right)}{1728 m^{2} c^{2}}, \\
& a_{0,6}=\frac{199745 n^{3}\left(n^{6}-3 n^{4}+3 n^{2}-1\right)}{162 c^{3}}, \\
& a_{0,8}=\frac{1445 n^{2}\left(n^{4}-2 n^{2}+1\right) m^{2}}{27 c^{4}}, \\
& a_{10,0}=-\frac{49 n\left(n^{2}-1\right)}{2 m} \text {, } \\
& a_{10,2}=-\frac{4 m}{c} \\
& a_{2,0}=-\frac{1}{1536 m^{7}}\left(79893275 m^{2} n^{15}-399466375 m^{2} n^{13}\right. \\
& +798932750 m^{2} n^{11}-798932750 n^{9} m^{2}+26244 \alpha^{2} c^{7} \\
& \left.+1536 m^{7} \alpha^{2}+399466375 m^{2} n^{7}-79893275 m^{2} n^{5}\right) \text {, }
\end{aligned}
$$

$$
\begin{aligned}
& a_{2,10}=-\frac{64 m^{5}}{81 c^{5}} \\
& a_{2,2}=-\frac{94325 n^{4}\left(n^{8}-4 n^{6}+6 n^{4}-4 n^{2}+1\right)}{64 c m^{3}}, \\
& a_{2,4}=\frac{1225 n^{3}\left(n^{6}-3 n^{4}+3 n^{2}-1\right)}{12 c^{2} m}, \\
& a_{2,6}=-\frac{17710 n^{2}\left(n^{4}-2 n^{2}+1\right) m}{27 c^{3}}, \\
& a_{2,8}=-\frac{760 m^{3} n\left(n^{2}-1\right)}{27 c^{4}}, \\
& a_{4,0}=-\frac{5187875 n^{4}\left(n^{8}-4 n^{6}+6 n^{4}-4 n^{2}+1\right)}{768 m^{4}}, \\
& a_{4,2}=\frac{18375 n^{3}\left(n^{6}-3 n^{4}+3 n^{2}-1\right)}{8 c m^{2}}, \\
& a_{4,4}=\frac{18725 n^{2}\left(n^{4}-2 n^{2}+1\right)}{18 c^{2}}, \\
& a_{4,6}=\frac{2920 m^{2} n\left(n^{2}-1\right)}{27 c^{3}}, \\
& a_{4,8}=\frac{80 m^{4}}{27 c^{4}}, \\
& a_{6,0}=-\frac{18865 n^{3}\left(n^{6}-3 n^{4}+3 n^{2}-1\right)}{48 m^{3}}, \\
& a_{6,2}=-\frac{4655 n^{2}\left(n^{4}-2 n^{2}+1\right)}{6 c m}, \\
& a_{6,4}=-\frac{1540 m n\left(n^{2}-1\right)}{9 c^{2}}, \\
& a_{6,6}=-\frac{160 m^{3}}{27 c^{3}} \\
& a_{8,0}=\frac{735 n^{2}\left(n^{4}-2 n^{2}+1\right)}{16 m^{2}}, \\
& a_{8,2}=\frac{115 n\left(n^{2}-1\right)}{c} \\
& a_{8,4}=\frac{20 m^{2}}{3 c^{2}} \\
& b_{0,0}=\frac{169785 n^{3}\left(n^{6}-3 n^{4}+3 n^{2}-1\right) c^{3}}{512 m^{6}}, \\
& b_{0,2}=\frac{17955 c^{3} n^{2}\left(n^{4}-2 n^{2}+1\right)}{128 m^{5}}, \\
& b_{0,4}=\frac{2835 c^{3} n\left(n^{2}-1\right)}{32 m^{4}} \text {, } \\
& b_{0,6}=-\frac{135 c^{3}}{8 m^{3}}
\end{aligned}
$$




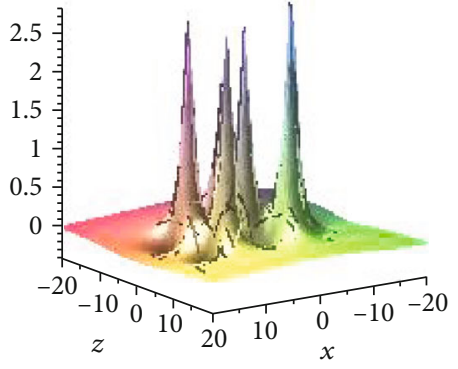

(a)

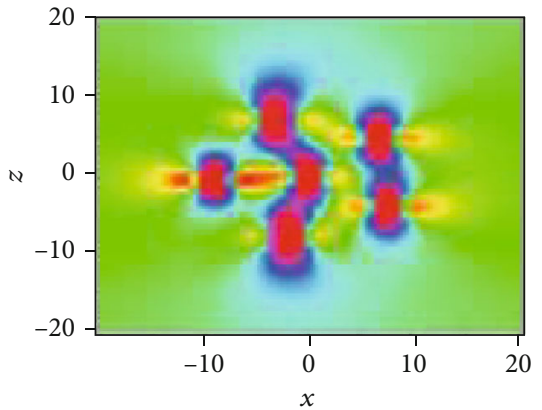

(b)

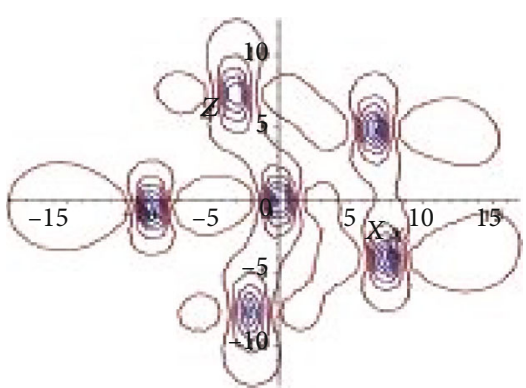

(c)

FIgUre 3: The 6-lump solution $u$ of Equation (1) with the parameter selections $m=-2, n=2, c=1, u_{0}=0, \alpha=80000, \beta=80000$. (a) Perspective view of the wave $u(X, Z)$, (b) overhead view of the wave $u(X, Z)$, and (c) the corresponding contour plot $u(X, Z)$.

$$
\begin{aligned}
& b_{2,0}=-\frac{2205 c^{2} n^{2}\left(n^{4}-2 n^{2}+1\right)}{64 m^{4}}, \\
& b_{2,2}=\frac{855 c^{2} n\left(n^{2}-1\right)}{8 m^{3}}, \\
& b_{2,4}=-\frac{45 c^{2}}{4 m^{2}}, \\
& b_{4,0}=-\frac{21 c n\left(n^{2}-1\right)}{8 m^{2}}, \\
& b_{4,2}=\frac{27 c}{2 m}, \\
& c_{0,0}=-\frac{12005 n^{3}\left(n^{6}-3 n^{4}+3 n^{2}-1\right)}{192 m^{3}}, \\
& c_{4,0}=-\frac{13 n\left(n^{2}-1\right)}{4 m^{2}}, \\
& c_{0,2}=-\frac{535 n^{2}\left(n^{4}-2 n^{2}+1\right)}{24 c m}, \\
& c_{2,0}=-\frac{245 n^{2}\left(n^{4}-2 n^{2}+1\right)}{16 m^{2}}, \\
& c_{0,4}=-\frac{5 m n\left(n^{2}-1\right)}{c^{2}}, \\
& c_{20 m^{3}}
\end{aligned}
$$

By this mean, we arrive at the 6-lump solution of Equation (1) represented by

$$
u(x, y, z, t, w)=(\ln f)_{X X}
$$

where $f$ is given in Equation (15). Figure 3 shows that the 3D plot of the 6-lump wave consists of a central peak and five 1lump waves in a ring. One can observe that the six peaks tend to the same height as $|\alpha|$ and $|\beta|$ increase.

\section{Conclusions}

In this work, we have analytically established and analyzed novel multiple lump solutions of a $(4+1)$-dimensional Fokas equation based on the bilinear equation and a new ansatz. A series of rational solutions including the 1-lump wave solutions, the 3-lump wave solutions, and the 6-lump wave solutions are obtained. The 1-lump wave has one positive peak and two negative peaks. In order to search the 3-lump and the 6-lump solutions, three polynomial functions $F_{n}, P_{n}$, and $Q_{n}$ are utilized. It is notable that these lump waves all have the properties $\lim _{x \rightarrow \pm \infty} u=u_{0}, \lim _{y \rightarrow \pm \infty} u=u_{0}$, and $\lim _{z \rightarrow \pm \infty} u=u_{0}$. The 3-lump and 6-lump waves consist of three and six independent single 1-lump waves, respectively. All the peaks of the multiple lump waves tend to the same height when $\alpha$ and $\beta$ are large enough. The results of this paper enrich the types of solutions of the $(4+1)$-dimensional Fokas equation. Comparing with the existing results in the literature, our results are new. We expect these results to provide some values for researching the dynamics of multiple waves in the deep ocean and nonlinear optical fibers. And it is very helpful for us to obtain the soliton molecules in the future.

\section{Data Availability}

No data were used to support this study.

\section{Conflicts of Interest}

The authors declare that they have no conflicts of interest.

\section{Acknowledgments}

The work is supported by the National Natural Science Foundation of China (project Nos. 11371086, 11671258, and 11975145), the Fund of Science and Technology Commission of Shanghai Municipality (project No. 13ZR1400100), the Fund of Donghua University, Institute for Nonlinear 
Sciences, and the Fundamental Research Funds for the Central Universities.

\section{References}

[1] W. Zhang, J. Zhou, X. Zhang, Y. Zhang, and K. Liu, "Quantitative investigation on force chain lengths during high velocity compaction of ferrous powder," Modern Physics Letters B, vol. 33, no. 10, article 1950113, 2019.

[2] S. Banerjee and L. Rondoni, Applications of Chaos and Nonlinear Dynamics in Science and Engineering, vol. 4, Springer, 2015.

[3] V. Gafiychuk, B. Datsko, and V. Meleshko, "Mathematical modeling of time fractional reaction-diffusion systems," Journal of Computational and Applied Mathematics, vol. 220, no. 1-2, pp. 215-225, 2008.

[4] I. R. Epstein, "Nonlinear oscillations in chemical and biological systems," Physica D: Nonlinear Phenomena, vol. 51, no. 1-3, pp. 152-160, 1991.

[5] K. Dysthe, H. E. Krogstad, and P. Müller, "Oceanic Rogue Waves," Annual Review of Fluid Mechanics, vol. 40, no. 1, pp. 287-310, 2008.

[6] L. Stenflo and M. Marklund, "Rogue waves in the atmosphere," Journal of Plasma Physics, vol. 76, no. 3-4, pp. 293-295, 2010.

[7] V. B. Efimov, A. N. Ganshin, G. V. Kolmakov, P. V. E. McClintock, and L. P. Mezhov-Deglin, "Rogue waves in superfluid helium," The European Physical Journal Special Topics, vol. 185, no. 1, pp. 181-193, 2010.

[8] M. Shats, H. Punzmann, and H. Xia, "Capillary Rogue Waves," Physical Review Letters, vol. 104, no. 10, article 104503, 2010.

[9] W. Liu and Y. Zhang, "Families of exact solutions of the generalized (3+1)-dimensional nonlinear-wave equation," Modern Physics Letters B, vol. 32, no. 29, article 1850359, 2018.

[10] H.-C. Ma and A.-P. Deng, "Lump solution of (2+1)-dimensional boussinesq equation," Communications in Theoretical Physics, vol. 65, no. 5, pp. 546-552, 2016.

[11] M. J. Ablowitz and H. Segur, Solitons and the Inverse Scattering Transform, Cambridge University Press, 1981.

[12] B. Guo, L. Ling, and Q. P. Liu, "Nonlinear Schrödinger equation: Generalized Darboux transformation and rogue wave solutions," Physical Review E, vol. 85, no. 2, article 026607, 2012.

[13] X. W. Yan, S. F. Tian, M. J. Dong, and L. Zou, "Bäcklund transformation, rogue wave solutions and interaction phenomena for a (3+1)-dimensional B-type Kadomtsev-PetviashviliBoussinesq equation," Nonlinear Dynamics, vol. 92, no. 2, pp. 709-720, 2018.

[14] M. O. Al-Amr, "Exact solutions of a family of higherdimensional space-time fractional KDV-type equations," Computer Science \& Information Technology, vol. 8, pp. 131141, 2018.

[15] A. F. Qasim and M. O. Al-amr, "Approximate solution of the Kersten-Krasil'shchik coupled Kdv-Mkdv system via reduced differential transform method," Eurasian Journal of Science \& Engineering, vol. 4, no. 2, p. 1, 2018.

[16] W. Cui Zhaqilao, "Multiple rogue wave and breather solutions for the (3+1)-dimensional KPI equation," Computers \& Mathematics with Applications, vol. 76, article 1099, no. 5, pp. 1099$1107,2018$.
[17] Y. Ohta and J. Yang, "Rogue waves in the Davey-Stewartson I equation," Physical Review E, vol. 86, no. 3, article 036604, 2012.

[18] T. Fang, H. Wang, Y.-H. Wang, and W.-X. Ma, "High-order lump-type solutions and their interaction solutions to a (3 +1 -dimensional nonlinear evolution equation," Communications in Theoretical Physics, vol. 71, no. 8, p. 927, 2019.

[19] X. Wang, S. Bilige, and J. Pang, "Rational solutions and their interaction solutions of the $(3+1)$-dimensional Jimbo-Miwa equation," Advances in Mathematical Physics, vol. 2020, Article ID 9260986, 18 pages, 2020.

[20] Y. Ohta and J. Yang, "General high-order rogue waves and their dynamics in the nonlinear Schrödinger equation," Proceedings of the Royal Society A: Mathematical, Physical and Engineering Sciences, vol. 468, no. 2142, pp. 1716-1740, 2012.

[21] A. S. Fokas, "Integrable nonlinear evolution partial differential equations in $4+2$ and $3+1$ dimensions," Physical Review Letters, vol. 96, no. 19, article 190201, 2006.

[22] A. S. Fokas, "Symmetries and integrability," Studies in Applied Mathematics, vol. 77, no. 3, pp. 253-299, 1987.

[23] Y. He, "Exact solutions for (4+1)-dimensional nonlinear Fokas equation using extended $F$-expansion method and its variant," Mathematical Problems in Engineering, vol. 2014, Article ID 972519, 11 pages, 2014.

[24] S. Zhang, C. Tian, and W. Y. Qian, "Bilinearization and new multisoliton solutions for the (4+1)-dimensional Fokas equation," Pramana, vol. 86, no. 6, pp. 1259-1267, 2016.

[25] Y. Li and H. Hu, "Nonlocal symmetries and interaction solutions of the Benjamin-Ono equation," Applied Mathematics Letters, vol. 75, pp. 18-23, 2018.

[26] M. Zhang and W. Cheng, "Recognition of mixture control chart pattern using multiclass support vector machine and genetic algorithm based on statistical and shape features," Mathematical Problems in Engineering, vol. 2015, Article ID 382395, 10 pages, 2015.

[27] S. T. Demiray and H. Bulut, "Investigation of dark and bright soliton solutions of some nonlinear evolution equations," ITM Web of Conferences, vol. 22, article 01056, 2018.

[28] L. Cheng and Y. Zhang, "Lump-type solutions for the (4 +1 )-dimensional Fokas equation via symbolic computations," Modern Physics Letters B, vol. 31, no. 25, article 1750224, 2017.

[29] M. O. Al-Amr and S. El-Ganaini, "New exact traveling wave solutions of the (4+1)-dimensional Fokas equation," Computers \& Mathematics with Applications, vol. 74, no. 6, pp. 1274-1287, 2017.

[30] S. Zhang and H.-Q. Zhang, "Fractional sub-equation method and its applications to nonlinear fractional PDEs," Physics Letters A, vol. 375, no. 7, pp. 1069-1073, 2011.

[31] H. Q. Sun and A. H. Chen, "Interactional solutions of a lump and a solitary wave for two higher-dimensional equations," Nonlinear Dynamics, vol. 94, no. 3, pp. 1753-1762, 2018.

[32] S. El-Ganaini and M. O. Al-Amr, "New abundant wave solutions of the conformable space-time fractional $(4+1)$-dimensional Fokas equation in water waves," Computers \& Mathematics with Applications, vol. 78, no. 6, pp. 2094-2106, 2019.

[33] W. J. Zhang and T. C. Xia, "Solitary wave, M-lump and localized interaction solutions to the (4+1)-dimensional Fokas equation," Physica Scripta, vol. 95, no. 4, article 045217, 2020 . 